\title{
Arterial Pulse Pressure Variation versus Central Venous Pressure as a Predictor for Fluid Responsiveness during Open Major Abdominal Operations
}

\author{
Mostafa M. Hussein, Raham H. Mostafa \\ Department of Anesthesia, Intensive Care and Pain Medicine, Faculty of Medicine, Ain Shams University, Cairo, Egypt \\ Email: mostafa_mansour361@hotmail.com,rahamhasan@yahoo.com
}

How to cite this paper: Hussein, M.M. and Mostafa, R.H. (2018) Arterial Pulse Pressure Variation versus Central Venous Pressure as a Predictor for Fluid Responsiveness during Open Major Abdominal Operations. Open Journal of Anesthesiology, 8, 43-53. https://doi.org/10.4236/ojanes.2018.82005

Received: January 21, 2018

Accepted: February 25, 2018

Published: February 28, 2018

Copyright $\odot 2018$ by authors and Scientific Research Publishing Inc. This work is licensed under the Creative Commons Attribution International License (CC BY 4.0).

http://creativecommons.org/licenses/by/4.0/

\begin{abstract}
Introduction: Fluid resuscitation is the cornerstone in the management of hemodynamically unstable patients. Dynamic parameters of fluid responsiveness, like pulse pressure variation, have the advantage of being more reliable index for fluid management. Objective: The aim of our study was to compare between arterial pulse pressure variation (PPV) versus central venous pressure (CVP) as a predictor for fluid responsiveness during major open abdominal operations. Patients and Methods: 60 adult patients under general anesthesia with mechanical ventilation underwent open major abdominal surgical procedures were included in our prospective randomized controlled study. Intravenous fluid was infused and monitored by CVP in control group or by PPV in the other group. Hemodynamic variables (heart rate, invasive blood pressure, PPV and CVP) were measured at baseline after anesthesia induction and every $10 \mathrm{~min}$, during first hour of operation, and then every $15 \mathrm{~min}$, till end of surgery. Blood loss and total i.v. fluid \& blood transfusion given to patients were recorded and compared between two groups intraoperatively. Results: Patients in the PPV group required more intraoperative fluid and blood transfusion than patients in CVP group to achieve more stable hemodynamic parameters. The fall in blood pressure ( $>20 \%$ of baseline) and increase in heart rate are more common in CVP group $(p<0.05)$. Conclusion: PPV is a better predictor and a good guide for fluid responsiveness. More stable hemodynamic variables are observed in PPV group.
\end{abstract}

\section{Keywords}

Central Venous Pressure, Pulse Pressure Variation, Fluid Responsiveness 


\section{Introduction}

Over the last decades, fluid therapy was considered as the first line of therapy during resuscitation of hemodynamically unstable patients. Although, fluid therapy increases cardiac output (CO) and improves blood pressure, volume overload can lead to pulmonary edema and interstitial edema which will increase morbidity and mortality [1] [2].

It was found that only one half of the critically ill patients are responsive to fluid therapy. Thus, fluid intake can be harmful when hemodynamically unnecessary. Accurate fluid prediction is crucial as it has a great impact on patient outcome. It is important to be able to predict increase in CO before fluid therapy is given. Clinical signs of volume depletion (e.g. heart rate, blood pressure, skin turgor and urine output) are routinely used but of limited sensitivity and specificity [3].

Numerous hemodynamic variables are studied to predict fluid responsiveness. Static variables (e.g. central venous pressure, pulmonary capillary wedge pressure, inferior vena cava diameter and left ventricle end diastolic volume) depend mainly on the preload estimation. These static variables are not reliable because Starling curves differ between patients. So, there may be a positive response at a given preload (preload dependency) or no response (preload independency) [4] [5] (Figure 1).

In contrast to static variables, dynamic variables (e.g. PPV and stroke volume variation, pleth variability index (PVI) and aortic blood flow measured by Doppler) are more reliable and more predictive for fluid responsiveness. These dynamic variables depend on the relation between cardiopulmonary interaction with mechanical ventilation. Mechanical ventilation produces cyclic changes in intrathoracic and trans-pulmonary pressures. These cyclic changes affect the left ventricle preload with resultant cyclic changes in left ventricle stroke volume and systemic arterial pressure in the preload dependent patients only [7] [8].

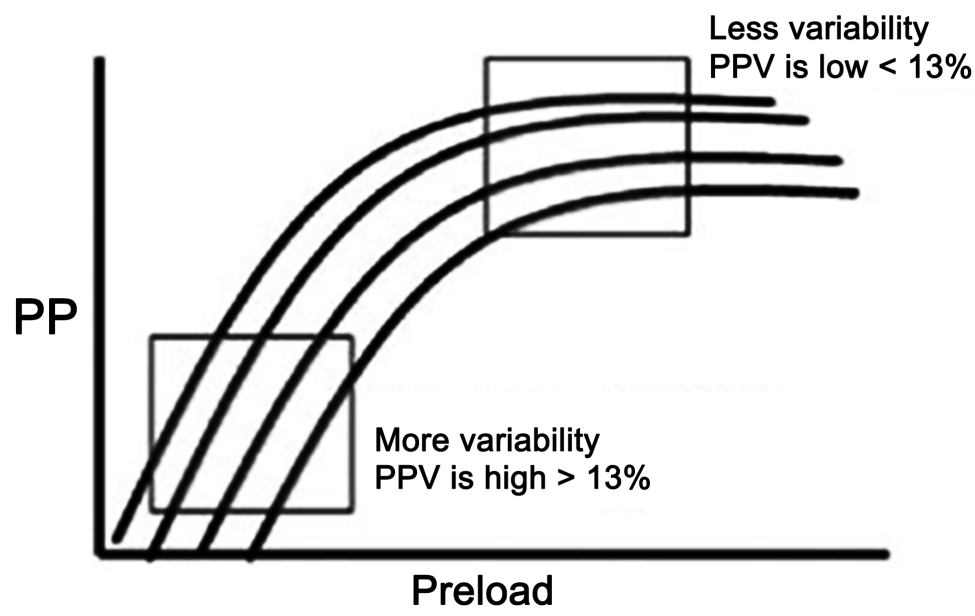

Figure 1. Prediction of patients' position on starling curve through arterial waveform analysis (PP: Pulse Pressure) [6]. 
PPV is calculated as the ratio between the maximum difference in pulse pressure (PP) observed over three respiratory cycles and the average of these two PPs as follows:

$$
\mathrm{PPV}=\left(\mathrm{PP}_{\max }-\mathrm{PP}_{\min }\right) /\left[\left(\mathrm{PP}_{\max }+\mathrm{PP}_{\min }\right) / 2\right] \times 100[9]
$$

Recently, PPV and stroke volume variation (SVV) indices can be continuously calculated and monitored through monitoring devices. IntelliVue MP Invigilator is a monitor that can continuously monitor PPV [10] [11].

Michard et al. [12] concluded that PPV more than 12\% - 13\% predicted fluid responsiveness in patients with septic shock or acute respiratory distress syndrome (ARDS) (Figure 2).

\section{Patients and Methods}

The study was conducted in the theatre of the general surgery department, at faculty of medicine, A in Shams university during the period from September 2014 to February 2016.

After approval of local ethical committee and taking informed written consent from patients, 60 adult patients, of both sexes, aged between 20 to 60 years, ASA I/II physical status, undergoing open major abdominal surgery under general anesthesia were enrolled in our study.

Exclusion criteria:

1) Age $<20$ years or $>60$ years.

2) Valvular heart disease or intra-cardiac shunts.

3) Arrhythmias.

4) Pregnancy or increased intra-abdominal pressure.

5) Chronic obstructive pulmonary disease.

6) Evidence of right ventricular failure.

Upon arrival to the operating room, $1 \mathrm{mg}$ midazolam was given intravenously to all patients as a premedication. Routine intraoperative monitoring for vital data (i.e. 5 leads ECG, arterial blood pressure, heart rate, capnography and pulse oximetry) was doneusing Philips MP 50 IntelliVue monitoring system (Philips Medical Systems, Böblingen, Germany). After local lidocaine infiltration, radial
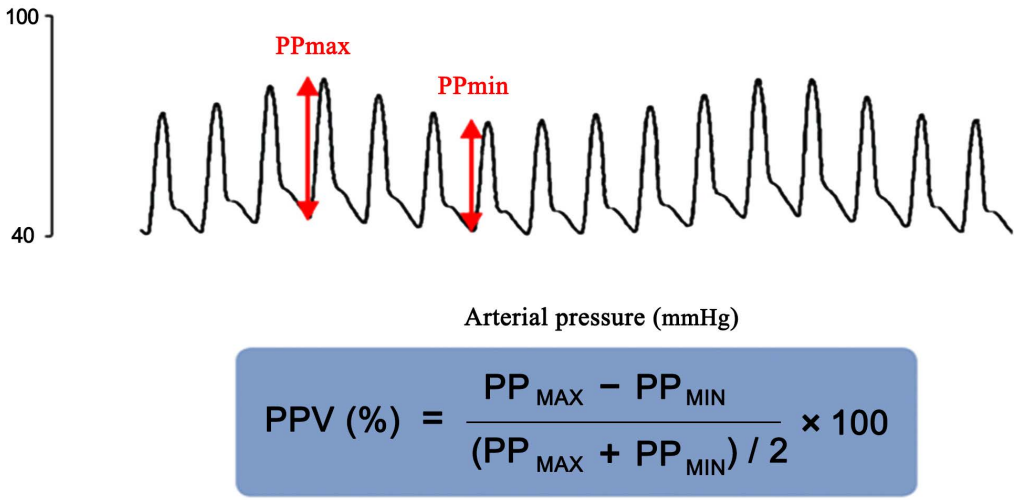

Figure 2. Method of calculation of PPV [13]. 
artery cannulation was done by $20 \mathrm{G}$ catheter. Pressure transducer was leveled at mid-axillary line and zeroed to atmospheric pressure. A series of hemodynamic variables are measured from the indwelling radial artery catheter including heart rate (beats/minute), systolic \& diastolic arterial pressure $(\mathrm{mmHg})$, and pulse pressure variation (PPV).

Induction of anesthesia was done using propofol $(2 \mathrm{mg} / \mathrm{kg})$, fentanyl $(2 \mu \mathrm{g} / \mathrm{kg})$, atracurium $(0.5 \mathrm{mg} / \mathrm{kg})$ with endotracheal intubation. Maintenance of anesthesia was done using sevoflurane $2 \%$ ( 1 minimal alveolar concentration) in oxygen/air gas mixture. Fentanyl $1 \mu \mathrm{g} / \mathrm{kg}$ bolus injection was given if required. Mechanical ventilation was maintained in volume control mode with tidal volume $8 \mathrm{ml} / \mathrm{kg}$, respiratory frequency 12 breaths/min. And positive end expiratory pressure (PEEP) $0 \mathrm{~cm} \mathrm{H}_{2} \mathrm{O}$ to maintain an $\mathrm{ET} \mathrm{CO}_{2}$ between $35-40 \mathrm{mmHg}$. Following induction of anesthesia, atriple-lumen central venous catheter (Certofix ${ }^{\oplus}$ Trio, B. Braun, Melsungen, Germany) was inserted in right internal jugular vein for central venous pressure (CVP) monitoring.

Patients who met the inclusion criteria were randomly allocated into either two groups (30 patients each) by computer generated program: CVP group (Control group) and $P P V$ group.

Lactated ringer and normal saline (crystalloids) were used alternatively to maintain normal CVP range $\left(5-10 \mathrm{~cm} \mathrm{H}_{2} \mathrm{O}\right)$ and to maintain $\mathrm{PPV}<13 \%$. In case of bleeding exceeding allowable blood loss, colloid and blood transfusion were given to maintain hemoglobin level around $10 \mathrm{gm} \%$ depending on patients' preoperative hemoglobin.

Systolic blood pressure (SBP), diastolic blood pressure (DBP), heart rate (HR), CVP (normal range $5-10 \mathrm{~cm} \mathrm{H}_{2} \mathrm{O}$ ) and PPV (normal range 10\% - 13\%) were measured at baseline after intubation, then every $10 \mathrm{~min}$. during first hour of surgery, then every $15 \mathrm{~min}$. Till end of surgery. The total volume of fluid (crystalloid \& colloid) and blood given to the patients during surgery were calculated.

In case of persistent hypotension (i.e. MAP $<65 \mathrm{mmHg}$ ) despite normal CVP and PPV, norepinephrine $0.1-0.7 \mu \mathrm{g} / \mathrm{kg} / \mathrm{min}$ was given.

\section{Sample Size Calculation}

Group sample sizes of 28 patients per group achieve $81 \%$ power to detect a difference of $500 \mathrm{ml}$ in blood loss between both groups assuming the mean blood loss in the control group is 2 liters with estimated group standard deviations of $650 \mathrm{ml}$ and with a significance level (alpha) of 0.05 using a two-sided two-sample $\mathrm{t}$-test. Thirty patients were included to replace any dropout.

\section{Statistical Analysis}

Data were analyzed using the Statistical Package for Social Sciences v 18 SPSS 18.0 for Windows (SPSS, Chicago, IL, USA). Normally distributed numerical data are presented as mean $\pm \mathrm{SD}$ and differences between groups were compared using the independent Student's $t$-test, data not normally distributed were compared using Mann-Whitney test and are presented as median (IQR) and cate- 
gorical variables were analyzed using the $\chi^{2}$ test or fisher exact test and are presented as number (\%). All $P$ values are two-sided. $p<0.05$ is considered statistically significant (Figure 3 ).

\section{Results}

Comparison of demographic data between the two groups showed no significant difference. The two groups were comparable in the general characteristics including age, sex, body weight, ASA, duration and type of surgery $(p>0.05)$ (Table 1).

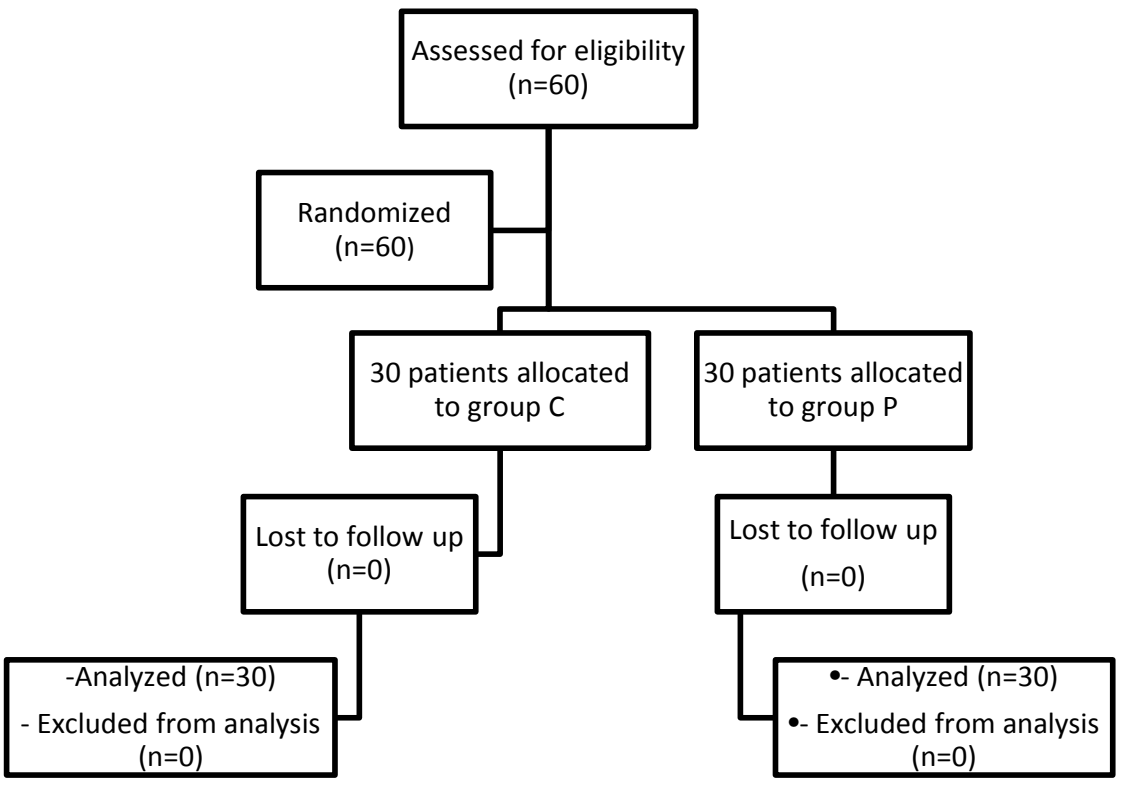

Figure 3. Flowchart showing the number of participants in each stage.

Table 1. Demographic data comparison among the two groups.

\begin{tabular}{cccc}
\hline Variables & Group $P(\mathbf{n}=30)$ & Group C $(\mathbf{n}=30)$ & $p$-value \\
\hline Age (yr.) & $51.1 \pm 11.23$ & $53.63 \pm 9.68$ & 0.353 \\
Gender (M/F) & $20 / 10$ & $16 / 14$ & 0.43 \\
ASA (I/II) & $18 / 12$ & $15 / 15$ & 0.604 \\
Body weight (Kg) & $88.77 \pm 12.4$ & $89.43 \pm 12.63$ & 0.837 \\
Duration of surgery (min.) & $178.23 \pm 57.64$ & $181.15 \pm 68.7$ & 0.843 \\
Type of surgery. & & & \\
-Partial gastrectomy & 5 & 1 & \\
-Total colectomy & 5 & 5 & \\
-Partial hepatectomy & 10 & 11 & \\
-Pancreatectomy & 3 & 1 & \\
-Right hemicolectomy & 2 & 1 & \\
-Wipple operation & 3 & 7 & \\
-Wedge hepatectomy & 1 & 0 & \\
- Sigmoidectomy & 1 & 3 & \\
-Abdomino-perineal & 0 & 1 & \\
Resection & & & \\
\hline
\end{tabular}

Values are expressed as mean $\pm \mathrm{SD}$ or number of patients. $p>0.05$ was considered statistically non-significant. 
No difference between the two groups as regards the preoperative $\mathrm{Hb}$. Level and the amount of intraoperative blood loss. The amount of crystalloid given intraoperatively was comparable in both groups $(p>0.05)$ (Table 2).

Group $P$ patients required more intraoperative colloid (i.e. $533.33 \pm 319.84$ ml.) than patients in group $\mathrm{C}$ who required only $239.73 \pm 166.67 \mathrm{ml}(p<0.001)$ (Table 2). The amount of intraoperative blood and fresh frozen plasma given are higher in group $P$ than group $C$. Intraoperatively, group $P$ patients received 1.97 \pm 0.81 units packed $\mathrm{RBC}$ and $1.77 \pm 0.73$ units FFP compared with $1.1 \pm 1.094$ units packed RBC and $1.1 \pm 1.03$ units FFP in group $\mathrm{C}(p<0.05)$ (Table 2).

Figures 4-6 showing comparison between the two groups regarding HR, SBP and DBP. Patients in CVP group are more tachycardic and hypotensive than patients in group $P(p<0.05)$. But, neither patients in the two groups required any vasopressor support.

Table 2. Intraoperative fluid management in the two groups.

\begin{tabular}{cccc}
\hline Variables & $\begin{array}{c}\text { Group } P \\
(\mathrm{n}=30)\end{array}$ & Group C (n=30) & $p$-value \\
\hline Preoperative Hb. (gm \%) & $11.28 \pm 1.057$ & $11.49 \pm 1.063$ & 0.468 \\
Intraoperative Blood Loss (ml.) & $1218.33 \pm 526.78$ & $1160 \pm 566.5$ & 0.681 \\
Intraoperative Crystalloid (ml.) & $2840 \pm 678.54$ & $2726.7 \pm 664.85$ & 0.516 \\
Intraoperative Colloid (ml.) & $533.33 \pm 319.84$ & $239.73 \pm 166.67^{*}$ & $<0.001$ \\
Intraoperative Blood (unit) & $1.97 \pm 0.81$ & $1.1 \pm 1.094^{*}$ & 0.001 \\
Intraoperative FFP. (unit) & $1.77 \pm 0.73$ & $1.1 \pm 1.03^{*}$ & 0.005 \\
CVP (cm $\left.\mathrm{H}_{2} \mathrm{O}\right)$ & $5.9 \pm 0.96$ & $6.3 \pm 0.92$ & 0.14 \\
$\mathrm{PPV}(\%)$ & $12.14 \pm 0.74$ & $12.37 \pm 0.93$ & 0.301 \\
\hline
\end{tabular}

Values are expressed as mean $\pm \mathrm{SD} .{ }^{*} p<0.05$ was considered statistically significant.

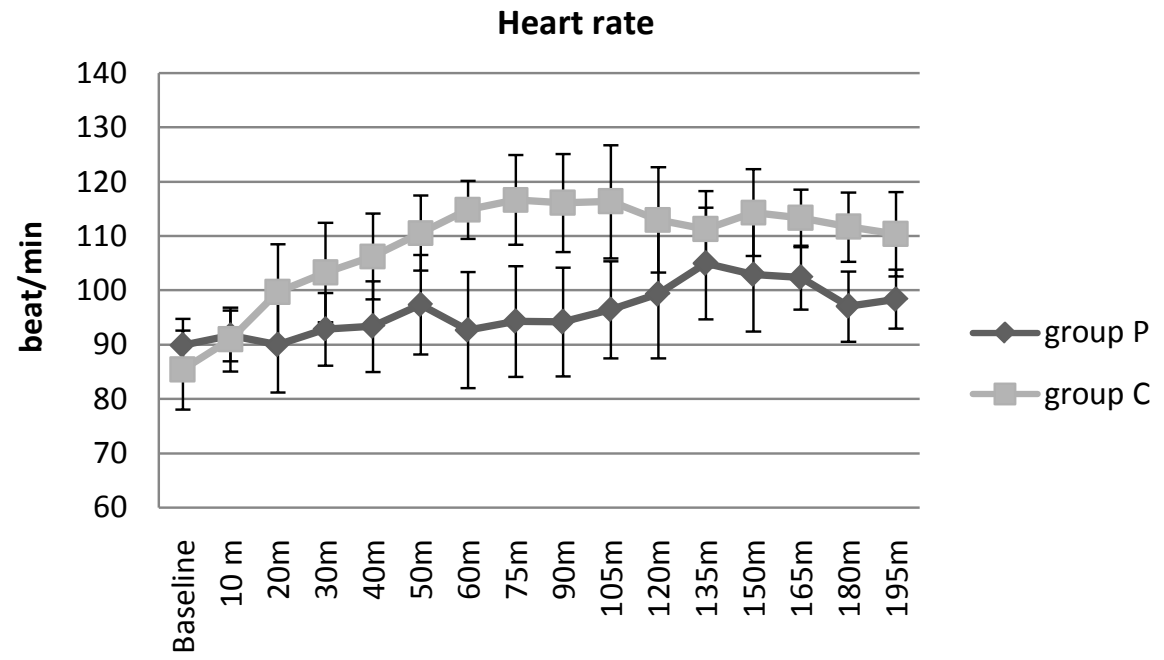

Figure 4. Heart rate comparison between the two groups. Data are expressed as mean \pm SD. $p<0.05$ is considered statistically significant. 


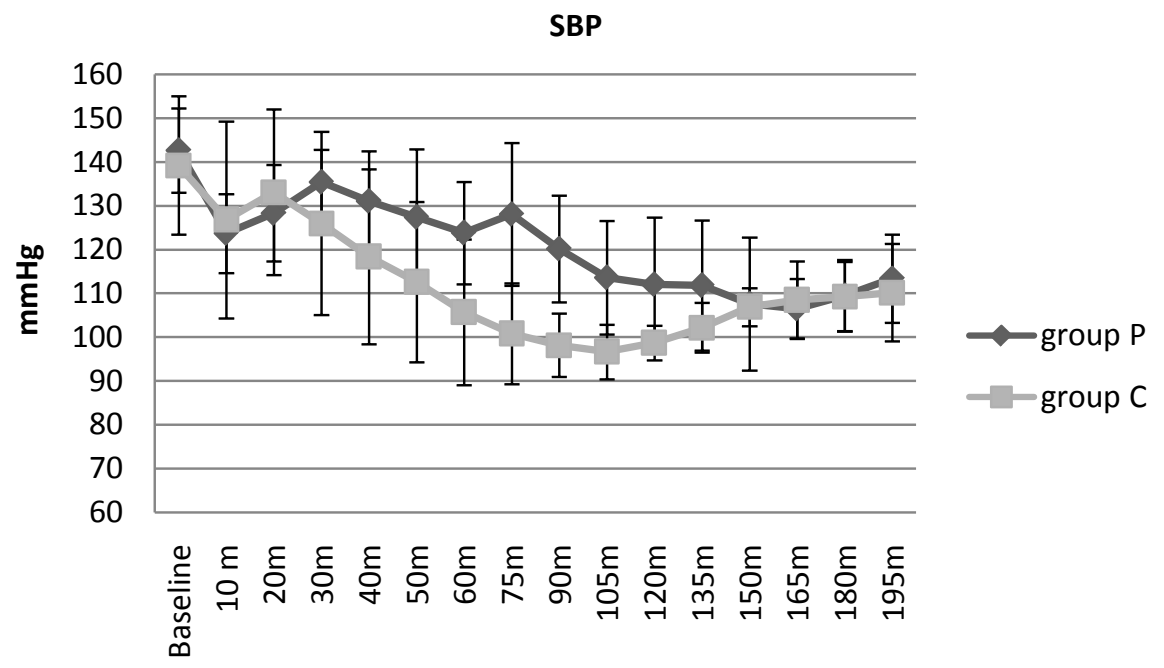

Figure 5. Systolic blood pressure (SBP) comparison between two groups. Data are expressed as mean \pm SD. $p<0.05$ is considered statistically significant.

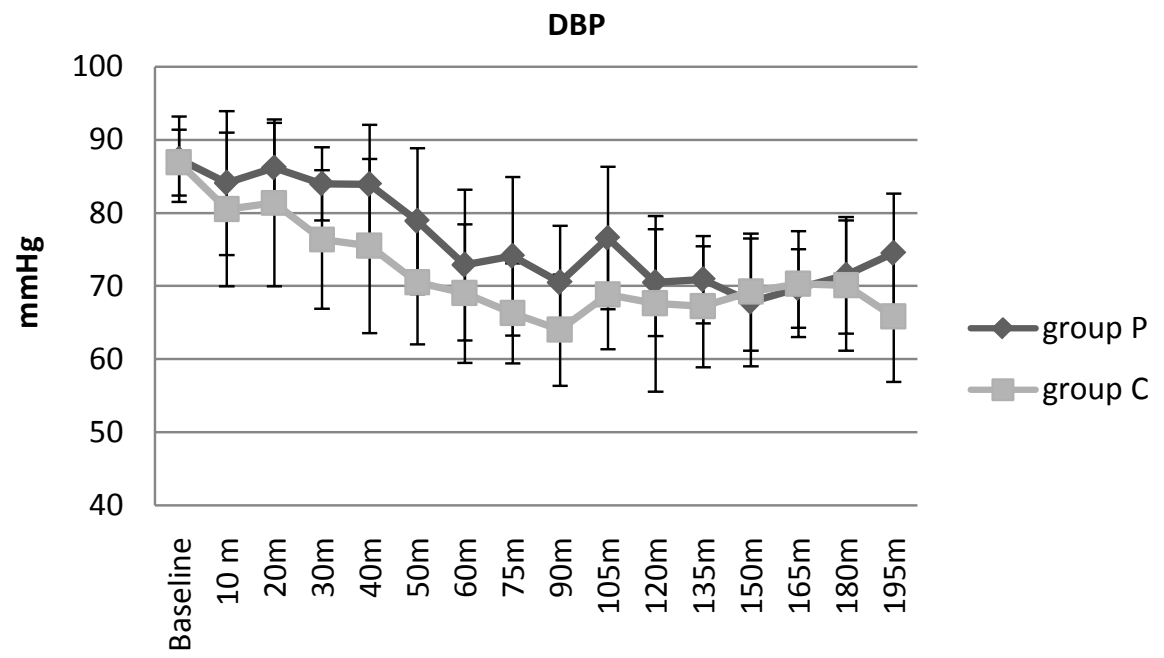

Figure 6. Diastolic blood pressure (DBP) comparison between two groups. Data are expressed as mean \pm SD. $p<0.05$ is considered statistically significant.

\section{Discussion}

In this study, patients in the PPV group received more intraoperative crystalloid but the difference between the two groups was insignificant. Colloid, blood and FFP transfusion were significantly more in the PPV group. Intraoperative blood loss was comparable between the two groups. Monitoring of vital data (i.e. HR, SBP, DBP) were done and were more stable in the PPV group. No patients in either groups required any norepinephrine infusion.

Assessment of volume requirement during major operations (e.g. Wipple operation) may be difficult due to use of irrigation fluids, blood loss beneath the drapes and inaccuracy of usual index for cardiac preload (i.e. CVP). So, fluid management should depend on the hemodynamics with volume adjustment guided by PPV. 
Pulse pressure variation measured by IntelliVue MP system possess the ability to predict fluid responsiveness in patients undergoing major operation with mechanical ventilation. This provides a useful guide for fluid management in these patients. Several clinical studies over the past years demonstrated the effectiveness of using PPV monitor for intraoperative and postoperative fluid guidance and how this can reduce the incidence of perioperative complications and hospital stay [14].

IntelliVue MP monitoring system can provide continuous automatic monitoring of PPV through analysis of the arterial pressure waveform without the need for special devices. Other methods for PPV monitoring can be used like PiCCO system and LiDCO system, but these methods require special device [15]. Kim et al. investigated the use of PPV in patients undergoing cardiac surgery and found good clinical application [16].

Fischer et al. demonstrated that the accuracy of PPV in predicting fluid requirement might be compromised in open chest operations. This is due to inaccuracy in reflection of phasic changes in preload and stroke volume, decreased cyclic changes in intrathoracic pressure and increased aortic impedance [17].

Despite the strong predictive value of PPV, Cannesson and colleagues [18] demonstrated that PPV may be inaccurate and inconclusive in predicting fluid responsiveness in $25 \%$ of patients under general anesthesia. Also, Biais and colleagues [19] used the term "gray zone" while investigating the clinical use of PPV. The practical value of this term allows determination of three zones: A zone with positive response, a zone with negative response and a third zone with uncertainty (i.e. gray zone). This zone occurs in patient receiving small tidal volume or those with low heart rate/respiratory rate ratio.

Gouvea et al. investigated the use of PPV during orthotopic liver transplantation. They found inability of PPV to predict fluid responsiveness during operation of liver transplantation due to decreased systemic vascular resistance (SVR), decreased heart function and operation stimulation [20].

A recent study done by Sundaram et al. investigated the use of PPV for intraoperative fluid management in adult patients undergoing craniotomy operation in supine and lateral position. They concluded that PPV monitoring resulted in better postoperative hemodynamic stability, avoided central line associated complications and reduced the additional cost [21].

There are many limitations for the use of PPV as a reliable predictor for fluid responsiveness. Tidal volume must be at least $8 \mathrm{ml} / \mathrm{kg}$ with controlled ventilation at fixed rate. This is because the low tidal volume ventilation cannot produce significant cyclic change in the intrathoracic pressure to induce preload variations. The cardiac rhythm must be sinus rhythm without any bradycardia or arrhythmia. PPV measurement is also influenced by the presence of spontaneous breathing [22]. PEEP must be avoided as it enhances cyclic changes in pleural pressures and hence increase PPV [23]. Also, drugs like $\beta$-blocker, norepinephrine and vasodilators interfere with PPV accuracy. Norepinephrine decreases 
PPV while vasodilators increase PPV [24].

Fluid resuscitation guided by CVP may leads to inaccurate volume replacement. CVP guided fluid therapy is effective only when the patients are on the ascending portion of the Frank-Starling curve, but when the left ventricle reach the flat portion of the curve, fluid intake will increase tissue edema and tissue dysoxia [3].

There were some limitations to our study: First, small sample size of the studied groups. Second, we did not measure the cardiac output which is the best method to differentiate between fluid responder from non-responder. Third, we did not continue monitoring the patients during the postoperative period. Colloid, like hydroxyethyl starch, was used during fluid resuscitation although evidence suggesting increased risk of nephropathy and coagulopathy associated with starch products [25].

\section{Conclusion}

PPV when combined with CVP can be a good guide to monitor fluid therapy in patients undergoing major abdominal operations.

\section{References}

[1] Brandt, S., Regueira, T., Bracht, H., Porta, F., Djafarzadeh, S., Takala, J., et al. (2009) Effect of Fluid Resuscitation on Mortality and Organ Function in Experimental Sepsis Models. Critical Care, 13, R186. https://doi.org/10.1186/cc8179

[2] Verdant, C. and De Backer, D. (2005) How Monitoring of the Microcirculation May Help Us at the Bedside. Current Opinion in Critical Care, 11, 240-244. https://doi.org/10.1097/01.ccx.0000158849.94225.11

[3] Michard, F. and Teboul, J.L. (2002) Predicting Fluid Responsiveness in ICU Patients: A Critical Analysis of the Evidence. Chest, 121, 2000-2008. https://doi.org/10.1378/chest.121.6.2000

[4] Marik, P.E. (2009) Techniques for Assessment of Intravascular Volume in Critically Ill Patients. Journal of Intensive Care Medicine, 24, 329-337. https://doi.org/10.1177/0885066609340640

[5] Sakka, S.G., Bredle, D.L., Reinhart, K. and Meier-Hellmann, A. (1999) Comparison between Intrathoracic Blood Volume and Cardiac Filling Pressures in the Early Phase of Hemodynamic Instability of Patients with Sepsis or septic shock. Journal of Critical Care, 14, 78-83. https://doi.org/10.1016/S0883-9441(99)90018-7

[6] Marik, P.E., Monnet, X. and Teboul, J.L. (2011) Hemodynamic Parameters to Guide Fluid Therapy. Annals of Intensive Care, 1, 1. https://doi.org/10.1186/2110-5820-1-1

[7] Pinsky, M.R. (2004) Using Ventilation-Induced Aortic Pressure and Flow Variation to Diagnose Preload Responsiveness. Intensive Care Medicine, 30, 1008-1010. https://doi.org/10.1007/s00134-004-2208-6

[8] Vieillard-Baron, A., Chergui, K., Augarde, R., Prin, S., Page, B., Beauchet, A., et al. (2003) Cyclic Changes in Arterial Pulse during Respiratory Support Revisited by Doppler Echocardiography. American Journal of Respiratory and Critical Care Medicine, 168, 671-676. https://doi.org/10.1164/rccm.200301-135OC

[9] Aboy, M., McNames, J., Thong, T., Phillips, C.R., Ellenby, M.S. and Goldstein, B. 
(2004) A Novel Algorithm to Estimate the Pulse Pressure Variation Index Delta PP. IEEE Transactions on Biomedical Engineering, 51, 2198-2203. https://doi.org/10.1109/TBME.2004.834295

[10] Cannesson, M., Slieker, J., Desebbe, O., Bauer, C., Chiari, P., Henaine, R., et al. (2008) The Ability of a Novel Algorithm for Automatic Estimation of the Respiratory Variations in Arterial Pulse Pressure to Monitor Fluid Responsiveness in the Operating Room. Anesthesia \& Analgesia, 106, 1195-1200. https://doi.org/10.1213/01.ane.0000297291.01615.5c

[11] Derichard, A., Robin, E., Tavernier, B., Costecalde, M., Fleyfel, M., Onimus, J., et al. (2009) Automated Pulse Pressure and Stroke Volume Variations from Radial Artery: Evaluation during Major Abdominal Surgery. British Journal of Anaesthesia, 103, 6786-6784. https://doi.org/10.1093/bja/aep267

[12] Michard, F., Boussat, S., Chemla, D., Anguel, N., Mercat, A., Lecarpentier, Y., et al. (2000) Relation between Respiratory Changes in Arterial Pulse Pressure and Fluid Responsiveness in Septic Patients with Acute Circulatory Failure. American Journal of Respiratory and Critical Care Medicine, 162, 134-138. https://doi.org/10.1164/ajrccm.162.1.9903035

[13] Lamia, B., Chemla, D., Richard, C. and Teboul, J.L. (2005) Clinical Review: Interpretation of Arterial Pressure Wave in Shock States. Critical Care, 9, 601-606. https://doi.org/10.1186/cc3891

[14] Cannesson, M., Musard, H., Desebbe, O., Boucau, C., Simon, R., Henaine, R. and Lehot, J.J. (2009) The Ability of Stroke Volume Variations Obtained with Vigileo/FloTrac System to Monitor Fluid Responsiveness in Mechanically Ventilated Patients. Anesthesia \& Analgesia, 108, 513-517. https://doi.org/10.1213/ane.0b013e318192a36b

[15] Cannesson, M., de Backer, D. and Hofer, C.K. (2011) Using Arterial Pressure Waveform Analysis for the Assessment of Fluid Responsiveness. Expert Review of Medical Devices, 8, 635-646. https://doi.org/10.1586/erd.11.30

[16] Kim, I.B., Bellomo, R., Fealy, N. and Baldwin, I. (2011) A Pilot Study of the Epidemiology and Associations of Pulse Pressure Variation in Cardiac Surgery Patients. Critical Care and Resuscitation, 13, 17-23.

[17] Fischer, M.O., Coucoravas, J., Truong, J., Zhu, L., Gérard, J.L., Hanouz, J.L. and Fellahi, J.L. (2013) Assessment of Changes in Cardiac Index and Fluid Responsiveness: A Comparison of Nexfin and Trans Pulmonary Thermodilution. Acta Anaesthesiologica Scandinavica, 57, 704-712. https://doi.org/10.1111/aas.12108

[18] Cannesson, M., Le Manach, Y., Hofer, C., Goarin, J.P., Lehot, J.J., Vallet, B., et al. (2011) Assessing the Diagnostic Accuracy of Pulse Pressure Variations for the Prediction of Fluid Responsiveness: A “Gray Zone” Approach. Anesthesiology, 115, 231-241. https://doi.org/10.1097/ALN.0b013e318225b80a

[19] Biais, M., Ehrmann, S., Mari, A., Conte, B., Mahjoub, Y., Desebbe, O., et al. (2014) Clinical Relevance of Pulse Pressure Variations for Predicting Fluid Responsiveness in Mechanically Ventilated Intensive Care Unit Patients: The Grey Zone Approach. Critical Care, 18, 587. https://doi.org/10.1186/s13054-014-0587-9

[20] Gouvea, G., Diaz, R., Auler, L., Toledo, R. and Martinho, J.M. (2009) Evaluation of the Pulse Pressure Variation Index as a Predictor of Fluid Responsiveness during Orthotopic Liver Transplantation. British Journal of Anaesthesia, 103, 238-243. https://doi.org/10.1093/bja/aep123

[21] Sundaram, S.C., Salins, S.R., Kumar, A.N. and Korula, G. (2016) Intra-Operative Fluid Management in Adult Neurosurgical Patients Undergoing Intracranial Tu- 
mour Surgery: Randomized Control Trial Comparing Pulse Pressure Variance (PPV) and Central Venous Pressure (CVP). Journal of Clinical and Diagnostic Research, 10, UC01-5. https://doi.org/10.7860/JCDR/2016/18377.7850

[22] Oliveira-Costa, C.D., Friedman, G., Vieira, S.R. and Fialkow, L. (2012) Pulse Pressure Variation and Prediction of Fluid Responsiveness in Patients Ventilated with Low Tidal Volumes. Clinics (Sao Paulo), 67, 773-778. https://doi.org/10.6061/clinics/2012(07)12

[23] Freitas, F.G., Bafi, A.T., Nascente, A.P., Assuncao, M., Mazza, B., Azevedo, L.C., et al. (2013) Predictive Value of Pulse Pressure Variation for Fluid Responsiveness in Septic Patients using Lung-Protective Ventilation Strategies. British Journal of Anaesthesia, 110, 402-408. https://doi.org/10.1093/bja/aes398

[24] Marik, P.E., Cavallazzi, R., Vasu, T. and Hirani, A. (2009) Dynamic Changes in Arterial Waveform Derived Variables and Fluid Responsiveness in Mechanically Ventilated Patients: A Systematic Review of the Literature. Critical Care Medicine, 37, 2642-2647. https://doi.org/10.1097/CCM.0b013e3181a590da

[25] Myburgh, J.A., Finfer, S., Bellomo, R., Billot, L., Cass, A., Gattas, D., et al. (2012) Hydroxyethyl Starch or Saline for Fluid Resuscitation in Intensive Care. New England Journal of Medicine, 367, 1901-1911. https://doi.org/10.1056/NEJMoa1209759 